\title{
Microcystic adnexal carcinoma simulating scarring alopecia*
}

\author{
Maria Christina Marques Nogueira Castanon ${ }^{1}$ \\ Raul Fernando Binato Lamim² \\ Camila Bastos Xavier Vassimon Silva ${ }^{1}$
}

\author{
Sandra Maria Gasparete Casali ${ }^{3}$ \\ Marina Vieira Nagahama ${ }^{1}$ \\ Rafael Guerra Nannetti de Carvalho ${ }^{1}$
}

DOI: http:/ /dx.doi.org/10.1590/abd1806-4841.20153445

\begin{abstract}
A bstract: The microcystic adnexal carcinoma is a rare, locally aggressive malignant adnexal neoplasm associated with significant morbidity. It is often underdiagnosed due to clinical and histopathological resemblance with other cutaneous neoplasms and / or a combination of lack of familiarity associated with inadequate samples. We report a case with clinical hypothesis of scarring alopecia and histopathological diagnosis of microcystic adnexal carcinoma with favorable outcome in a follow-up of eleven years, after surgical treatment.
\end{abstract}

Keywords: Alopecia; Pathology; Skin neoplasms

\section{INTRODUCTION}

Adnexal cutaneous tumors represent a diverse and broad spectrum of tumors originated from hair, sebaceous glands, apocrine and eccrine glands, encompassing numerous subgroups of both benign and malignant appendicular neoplastic processes. ${ }^{1}$

Microcystic Adnexal Carcinoma (MAC) is a malignant adnexal cutaneous neoplasm, rare, with follicular differentiation, indolent, locally aggressive, of invasive pattern and destructive, rarely evolving with metastases and easily mistaken, clinically and histologically, for other cutaneous neoplasms, both benign and malignant. ${ }^{1}$

Due to its innocuous clinical appearance and slow progression, a delay between onset of lesion and diagnosis is frequently observed. ${ }^{2}$

We present a clinical case of MAC with initial diagnostic hypothesis of scarring alopecia.

\section{CASE REPORT}

Female patient, white, 67 years old, presenting a whitish hardened plaque, with a smooth and shiny surface, without hairs, painless and measuring about
$5 \mathrm{~cm}$ in diameter in the occipital region (Figure 1). Such findings led to a clinical hypothesis of scarring alopecia. Initially, the histopathological report of the fragment obtained by punch biopsy was a neoplastic process of epithelial lineage, infiltrative and with syringomatoid differentiation. ${ }^{3}$ Later, histopathology of the surgical piece, after augmented excision of the tumor, revealed an infiltrative neoplastic process with areas of syringomatoid differentiation and areas of follicular differentiation, suggesting low degree Microcystic Adnexal Carcinoma infiltrating dermis and hypodermis, with compromised surgical margins (Figures 2, 3 and 4). A third augmented excision, with the confection of cutaneous flap, was performed and it still presented residual neoplasm, although with free surgical margins. A fourth reparative surgery was necessary for the reconstitution of extensive and deep losses of tissue in the scalp, with bone exposure resulting from previous augmented resection of the tumor. Postoperative evolution was satisfactory and the patient remains asymptomatic, without relapse of the disease in eleven years of clinical follow-up.

Received on 12.02.2014

Approved by the Advisory Board and accepted for publication on 29.03.2014

Work performed in partnership with: Universidade Federal de Juiz de Fora, private dermatology clinic and Pathology laboratory/Charity Hospital (Santa Casa de Misericórdia) in Juiz de Fora (MG), Brazil.

Financial Support: No charge.

Conflict of Interest: None.

Universidade Federal de Juiz de Fora (UFJF) - Juiz de Fora (MG), Brazil.

Santa Casa de Misericórdia de Juiz de Fora - Juiz de Fora (MG), Brazil.

Private clinic - Juiz de Fora (MG), Brazil.

(C2015 by Anais Brasileiros de Dermatologia 


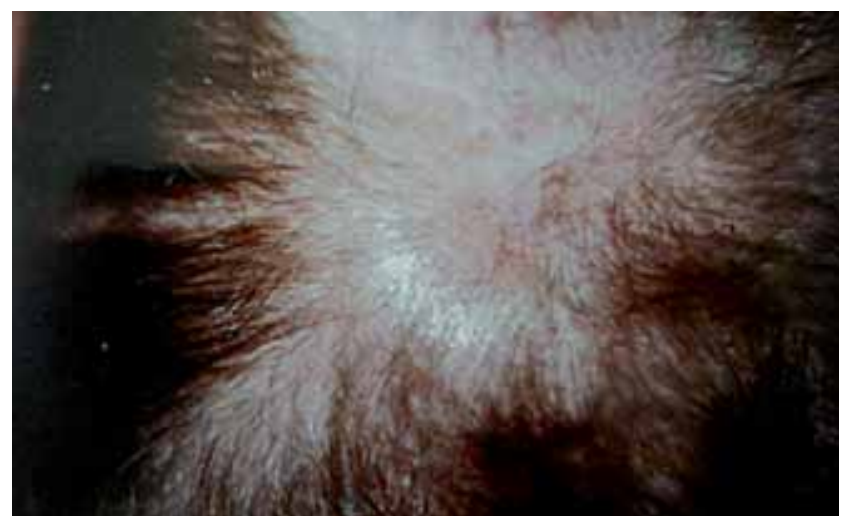

FIGURE 1: Hardened plaque with smooth and shiny surface, without hairs, measuring $5 \mathrm{~cm}$ in diameter located in the occipital region

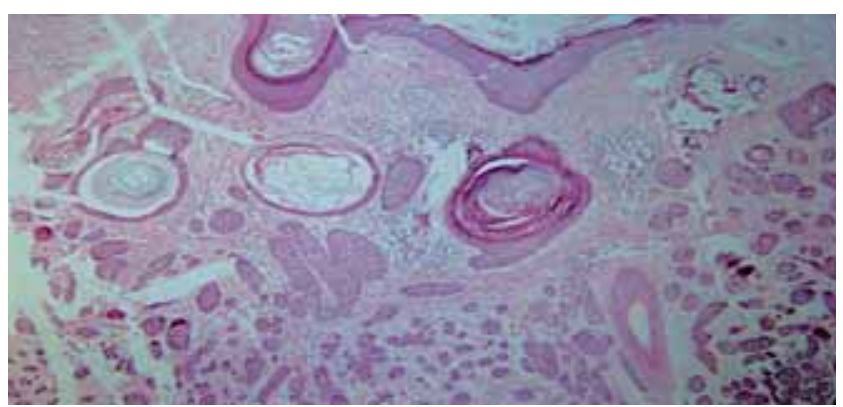

Figure 2: Nodular aggregates and epithelial cell cords associated with corneal microcysts in the most superficial dermis. H\&E 100X

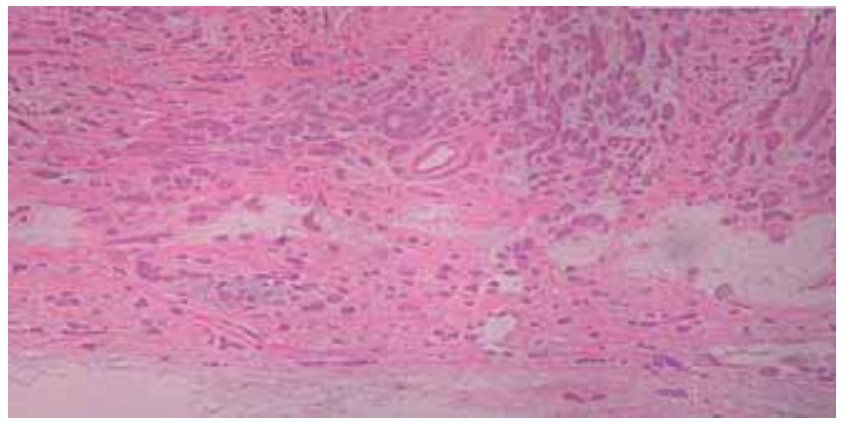

Figure 3: Cell aggregates and epithelial cords, surrounded by dense fibrous stroma, infiltrating the deep dermis and subcutaneous cell tissue. Observe the absence of pilosebaceous units. H\&E 40X

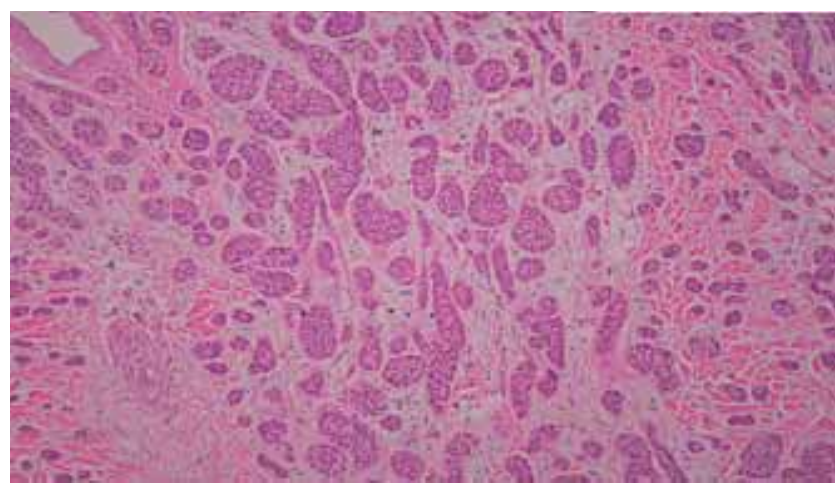

FIGURE 4: Infiltrative pattern, dense fibrous stroma and absence of nuclear pleomorphism. Observe the syringomatoid aspect, hence the synonymy "syringomatous carcinoma" H\&E 100X

\section{DISCUSSION}

The study of epidemiological characteristics and natural history of MAC is difficult in light of the rarity of this neoplasm. ${ }^{3}$ It predominates in females and usually manifests itself between the $4^{\text {th }}$ and $6^{\text {th }}$ decade of life, although there are reports of occurrences in children. ${ }^{4,5}$ It occurs more frequently in white patients, being rare in blacks. It demonstrates predilection for the head, particularly the periorbital and nasolabial regions. ${ }^{3}$ Lesions on the neck, scalp and orbit are rare. ${ }^{6,7}$

Its etiology is unknown, with evidences that therapeutic cutaneous irradiation, UV radiation and immunodeficiency are predisposing factors. ${ }^{4}$

The typical clinical presentation is a plaque or a poorly-defined and hardened nodule, solitary, skin colored to yellowish, smooth surface, rarely ulcerated, of slow growth and usually painless, measuring 0.5 to $2 \mathrm{~cm}$, but reaching up to $12 \mathrm{~cm}$ in its largest diameter. ${ }^{2}$ In general it is asymptomatic, but it can manifest pain, burning, stinging sensation, anesthesia or paresthesia arising from perineural invasion. It is locally aggressive, with potential of tissue destruction; metastases have been reported. 3,6

Histologically it is infiltrative and poorly defined, presenting glandular and follicular differentiation. Cords and nests of squamous and basal cells of benign aspect as well as keratocysts are observed in the papillar and medium dermis. As it invades the reticular dermis it presents ductal structures similar to glands, covered by one to two layers of flat or cuboidal cells, with penetration of perineural spaces, skeletal muscle and adipose tissue. The stroma is dense, fibrous and of sclerotic appearance in infiltrative areas. ${ }^{7}$

Nests and ductal structures may present appendages similar to tadpole tails, seen in the syringoma and in the desmoplastic trichoepithelioma. Eccrine glandular differentiation is more common, but apocrine differentiation may also occur. ${ }^{7}$ Cytologic atypia and mitoses are rare, being frequent in recurrent tumors, which is probably associated with a more aggressive biological behavior.8

The immunohistochemical pattern consists in the expression of carcinoembryonic antigen (CEA), epithelial membrane antigen (EMA) and cytokeratin cocktail (AE1/AE3), sustaining follicular and eccrine differentiation. Backing the follicular differentiation, the tumor expresses hard cytokeratin (AE13 and AE14) ${ }^{7,8}$ It presents a low proliferation index in immunolabeling with MIB. ${ }^{1,9}$ Occasionally there is immunoreactivity for hormone receptors, which requires thorough clinical investigation to exclude breast carcinoma. ${ }^{3}$

Histopathological differential diagnosis includes trichoadenoma, syringoma, desmoplastic 
trichoepithelioma, sclerodermiform basal cell carcinoma, desmoplastic squamous cell carcinoma and metastatic breast carcinoma. ${ }^{7}$

The therapeutic modalities employed include radiotherapy, simple excision and Mohs micrographic surgery. ${ }^{4}$

Long-term follow-up is recommended due to the elevated rates of recurrence. Overall survival is satisfactory. The majority of recurrences occur on average in the first 3 years, however there are reports of relapse in up to 30 years after surgery. ${ }^{10}$
In the case reported, the initial clinical hypothesis of scarring alopecia is justified by the locally aggressive biological behavior of MAC causing the destruction of pilosebaceous follicles and the dense fibrous stroma mimicking a scarring process, besides the uncommon topography. The inclusion of MAC in the differential clinical diagnosis of scarring alopecias is also suggested, in spite of being a remote possibility. We also emphasize the importance of a representative incisional biopsy covering the depth of the lesion, providing a correct initial diagnosis and consequently a less mutilating and definitive treatment, avoiding relapses. $\square$

\section{REFERENCES}

1. Goldstein DJ, Barr RJ, Santa Cruz DJ. Microcystic adnexal carcinoma: A distinct clinicopathologic entity. Cancer. 1982;50:566-72.

2. Abbate M, Zeitouni NC, Seyler M, Hicks W, Loree T, Cheney RT. Clinical course, risk factors, and treatment of microcystic adnexal carcinoma: a short series report. Dermatol Surg. 2003;29:1035-8.

3. Rotter N, Wagner H, Fuchshuber S, Issing WJ. Cervical metastases of microcystic adnexal carcinoma in an otherwise healthy woman. Eur Arch Otorhinolaryngol. 2003;260:254-7

4. Leibovitch I, Huilgol SC, Selva D, Lun K, Richards S, Paver R. Microcystic adnexal carcinoma: treatment with Mohs micrographic surgery. J Am Acad Dermatol. 2005;52:295-300.

5. Callahan EF, Vidimos AT, Bergfeld WF. Microcystic adnexal carcinoma (MAC) of the scalp with extensive pilar differentiation. Dermatol Surg. 2002;28:536-9.

6. Clement $\mathrm{Cl}$, Genge J, O'Donnell BA, Lochhead AG. Orbital and periorbital microcystic adnexal carcinoma. Ophthal Plast Reconstr Surg. 2005;21:97-102.

7. Chiller K, Passaro D, Scheuller M, Singer M, McCalmont T, Grekin RC. Microcystic adnexal carcinoma: forty-eight cases, their treatment, and their outcome. Arch Dermatol. 2000;136:1355-9.

8. Ongenae KC, Verhaegh ME, Vermeulen AH, Naeyaert JM. Microcystic adnexal carcinoma: an uncommon tumor with debatable origin. Dermatol Surg. 2001;27:979-84

9. LeBoit PE, Sexton M. Microcystic adnexal carcinoma of the skin. A reappraisal of the differentiation and differential diagnosis of an under recognized neoplasm. J Am Acad Dermatol. 1993;29:609-18.

10. Yu JB, Blitzblau RC, Patel SC, Decker RH, Wilson LD. Surveillance, Epidemiology, and End Results (SEER) database analysis of microcystic adnexal carcinoma (sclerosing sweat duct carcinoma) of the skin. Am J Clin Oncol. 2010;33:125-7.

\author{
M AILING ADDRESS: \\ $M$ aria Christina $M$ arques $N$ ogueira $C$ astañon \\ D epartamento de $\mathrm{M}$ orfologia - ICB \\ U niversidade Federal de Juiz de Fora \\ Rua J osé Lourenço Kelmer, s/n - Campus U niversitário \\ Bairro São Pedro \\ 36036-900 - Juiz de Fora - M G \\ Brazil \\ E-mail: christina.nogueira@ufjf.edu.br
}

H ow to cite this article: Castanon MCMN, Casali SMG, Lamim RFB, Nagahama MV, Silva CBXV, Carvalho RGN. Microcystic adnexal carcinoma simulating scarring alopecia. An Bras Dermatol. 2015;90 (3 Suppl 1): S36-8. 